\title{
Mouse paw preference: effects of variations in testing protocol
}

\author{
M. Barbara Bulman-Fleming a,*, M. Philip Bryden ${ }^{1,}$, , Timothy T. Rogers ${ }^{b}$ \\ a Department of Psychology, University of Waterloo, Waterloo, Ontario N2L 3G1, Canada \\ ${ }^{\mathrm{b}}$ Department of Psychology and the Neural Processes in Cognition Program, Center for the Neural Basis of Cognition, \\ Carnegie-Mellon University Pittsburgh, Pittsburgh, Pennsylvania, USA
}

Received 24 May 1996; received in revised form 11 October 1996; accepted 11 October 1996

\begin{abstract}
Two experiments using two inbred strains of mice were conducted to study mouse paw preference. In the first study, 250 $(50 \times 5)$ paw reaches from each of 12 male and 12 female C57BL/6J mice were observed over an 8-week period using the Collins paw preference apparatus to investigate questions relating to the independence of reaches, the size of the tube into which the animals reach for flakes of food, and practise effects. Animals appeared to be much more highly lateralized when two independent reaches per day for 25 days (I protocol) were observed than when 50 reaches were observed in a single session ( $\mathrm{S}$ protocol). Paradoxically, however, we found no evidence for the lack of independence of reaches when we examined the sequences of reaches performed by animals under the $\mathrm{S}$ protocol conditions. With practise, animals became slightly more lateralized, but there were no effects of sex, and approximately equal numbers of mice were left-pawed as were right-pawed. The size of the tube had no effect. The second study used 30 BALB mice, each of which was tested with both the I and S protocols, with order of testing counterbalanced. There was a significant protocol $\times$ order interaction such that those doing $\mathrm{S}$ first were more highly lateralized on the I task, but those doing I first were highly lateralized on both tasks. (C) 1997 Elsevier Science Ireland B.V.
\end{abstract}

Keywords: Inbred strains; Independent reaches; Lateralization; Mouse paw preference

\section{Introduction}

The relations among handedness and various measures of hemispheric specialization in humans are complex; therefore, it is not surprising that despite much research having been carried out concerning these topics over the past 25 years, there are many questions yet to be answered. The relatively recent discovery of laterality effects in many non-human species has opened up new possibilities for studying the nature and development of lateralization of function [1-3]. Because of the amount of information known about their genetics and their short gestation period, mice are ideal subjects for investigations of this sort, and may well provide the

\footnotetext{
* Corresponding author. Tel: + 15198884567 (ext. 3043); fax: + 1 519 7468631; email: bfleming@watarts.uwaterloo.ca

${ }^{1}$ Phil Bryden died suddenly of a heart attack in August, 1996, at the age of 61 . This paper is dedicated to his memory.
}

clues for unravelling the genetics of hemispheric specialization $[4,5]$.

Collins has developed a technique to assess the handedness, or pawedness, of mice wherein animals reach into a tube for food with either one or the other paw. The number of right paw entries (RPE score) is a measure of the degree and direction of paw preference of the animal, and can easily be converted to a metric of degree of preference that is independent of direction (the preferred paw entry score, or PPE). He has reported that the paw preference test yields measures of lateralized behaviour that are stable over time, and do not appear to be task specific [6]. He has also shown that degree of preference is sensitive to genetic selection [7,8]; mice of the Collins HI strain are more lateralized than those of the Collins LO strain. Though mice show individual asymmetry on this task, most workers have reported equality of the distribution of asymmetric 
forms at the population level $[8,9$; see also 10]. Recently, however, Waters and Denenberg [11] reported a significant leftward bias on the Collins test in a large number of mice $(n=693)$, as well as significant strain differences in direction of laterality in the Collins task. This prompted them to reanalyze several earlier studies, in the course of which they found significant or near-significant leftward population biases in all the studies they examined. Unpublished data from our laboratory also support these results [12]. Be that as it may, as Collins himself has pointed out, the usefulness of the mouse handedness model is not predicated on the similarity of its distribution of handedness types to those of other species [8]. Biddle's group [13] has also recently reported strain differences in RPE scores and our own independent reanalysis of the Signore et al. data supports both a leftward population bias and strain differences in $\mathrm{RPE}^{2}$.

The Collins test has been a very popular method for determining paw preference in mice; several groups of workers have employed this procedure to investigate the influences of various factors on mouse paw preference [14-19,11]. As a result of this popularity and of the recent results mentioned above, which suggest that the mouse model for human handedness may be even more relevant than was previously believed, it would seem important to investigate the parameters of the paw preference testing protocol. The usual protocol for the Collins test involves recording 50 paw reaches of each animal while observing several animals during a testing session. Because more than one of the animals often reach at the same time, it is not possible to record the animals' first 50 paw reaches. Furthermore, the individual reaches may not be statistically independent of one another.

We had several questions concerning the Collins procedure that prompted the studies we report here. We studied: (1) to what extent a successful previous reach would facilitate subsequent reaches with the same paw; (2) whether the size of the tube into which the animals reach for food would affect the degree of paw preference; (3) the degree to which the animals become more lateralized with practise; and (4) if measures based on the 50 (non-consecutive) paw reaches that are normally recorded would correlate highly with those derived from the first 50 reaches.

\footnotetext{
${ }^{2}$ A reanalysis (using loglinear procedures) of the Signore et al. data from their Fig. 1 reveals that significantly more mice were left- than right-pawed in that study $(P=0.01, n=450)$ and suggests that there may be directional differences among the 11 that were tested $(P=0.06)[9]$.
}

\section{Study 1}

\subsection{Methods}

\subsubsection{Subjects}

The 12 male and 12 female mice used were offspring of $\mathrm{C} 57 \mathrm{BL} / 6 \mathrm{~J}$ mice obtained from the Jackson Laboratory, Bar Harbor, ME. They were placed in standard plastic mouse cages with same-sex siblings from the time of weaning and had free access to tap water and lab chow (Purina Lab Chow 5001) until the beginning of the study, when they were approximately 10 weeks old.

\subsubsection{Paw preference testing sessions}

2.1.2.1. SN1. In the late afternoon before the first testing session, the lab chow was removed from the food hoppers in order to motivate the animals to learn the reaching task the next morning. Four animals at a time were placed into the apparatus pictured in Fig. 1 and were observed reaching for flakes of rolled oat cereal until 50 reaches were recorded for each animal. (Details of the apparatus can be found in [14].) The internal diameter of the food tube for this session was $6.5 \mathrm{~mm}$ (narrow), the same as that used by Collins [8]. This session was videotaped.

2.1.2.2. SN1V. Data for this session are those obtained from the analysis of the videotapes of session SN1. Therefore, they represent the first 50 reaches of each animal during the SN1 session.

2.1.2.3. IND. This independent-reaches session lasted for 5 weeks. Every weekday for 5 weeks each animal was placed by itself into the apparatus (which was configured with the narrow tubes) on two separate occasions (separated by at least $10 \mathrm{~min}$ ), and the first paw reach was noted. Because the animals had learned the task during the first session, it was usually not necessary to deprive them of food in order for them to reach during this or subsequent sessions. Several of the animals did not reach on one day, and there was one animal who did not reach on two days. These animals were food-deprived for the next day's testing, and their missing reaches were made up at the end of the 5-week testing period.

2.1.2.4. SN2. This took place during the week following the completion of the IND session, and was identical to SN1 except that it was not recorded on videotape. For animals that took a long time to reach on the independent-reaches trials, the food was taken away the afternoon before the test. The other animals were not food-deprived. 


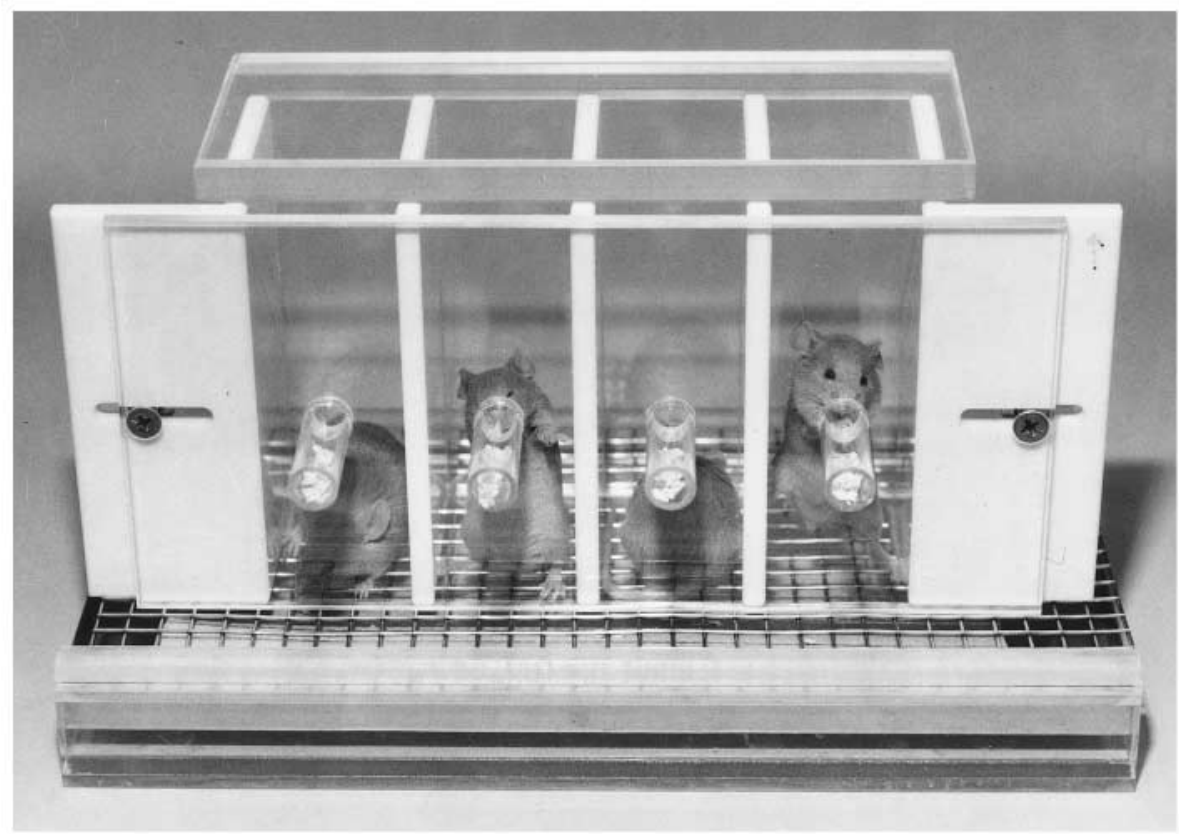

Fig. 1. The mouse paw preference testing apparatus (from [6]). The paw with which the mouse makes each of 50 reaches for food is recorded by an observer. Reprinted from Ref. [6] (with kind permission from Elsevier Science B.V.).

2.1.2.5. $S W$. The feeding tube's inner diameter for this session, which took place 2 days after session $\mathrm{SN} 2$, was wider than that for the other three sessions $(9 \mathrm{~mm})$. Animals were not food-deprived for this test.

\subsection{Results}

The right paw entry (RPE) score is the number of times, in a session of 50 observed reaches, that an animal reaches into the glass tube with its right paw. The preferred paw entry (PPE) score is then simply |RPE-25|, and ranges between 0 (a mouse using the left paw as many times as the right) and 25 (either 50 or 0 reaches with the right paw). The RPE thus is a measure of the direction of paw preference whereas the PPE measures the degree of paw preference.

Preliminary results indicated no effects of sex, so male and female data were pooled for subsequent analyses.

\subsubsection{Testing protocol}

The question of the statistical independence of paw reaches for the standard Collins protocol was investigated by performing a one-sample runs test for randomness [20] on the SN1V data, the videotaped data from session SN1. Only four of the 24 animals showed significant runs effects. Two of these shifted from one paw to the other more often than would be expected by chance, whereas two tended to perseverate in their responses. This general pattern provides little support for the notion that animals tend to repeat successful reaches.

\subsubsection{Paw preference}

No mouse reached exactly the same number of times with its right as with its left paw in any of the sessions. Classifying a mouse with an RPE score of less than 25 as left-pawed and one with an RPE score of greater than 25 as right-pawed, there were roughly equal numbers of mice classified as left- and as right-pawed for each session. Mean RPE and PPE scores, as well as the numbers of mice classified as right- and left-pawed for each session are shown in Table 1. In general, the direction of preference remained consistent from session to session. Seventeen animals were consistent across all sessions; three differed only on the SW session, three differed in the $\mathrm{SN} 1$ session, and one in the I session. In the SN1 session, classifications by observation (SN1) and by videotape (SN1V) were consistent in every case.

Table 1

Study 1 means for raw RPE and PPE scores and numbers of mice classified as right- and left-pawed ( $n=24 \mathrm{C} 57 \mathrm{BL} / 6 \mathrm{~J}$ mice)

\begin{tabular}{llll}
\hline Session & RPE (S.D.) & PPE (S.D.) & R/L classification \\
\hline SN1 & $26.4(17.7)$ & $15.1(8.9)$ & $13 / 11$ \\
SN1V & $26.8(19.3)$ & $17.3(8.1)$ & $13 / 11$ \\
IND & $23.5(23.5)$ & $22.6(4.4)$ & $11 / 13$ \\
SN2 & $24.6(19.7)$ & $17.5(8.2)$ & $10 / 14$ \\
SW & $25.2(20.2)$ & $18.3(7.6)$ & $11 / 13$ \\
\hline
\end{tabular}

$\mathrm{SN} 1$, first standard-protocol session with narrow feeding tube; SN1V, videotaped data of first 50 reaches from SN1; IND,

reaches session; SN2, second standard-protocol session, narrow tube; $\mathrm{SW}$, final standard-protocol session, wider tube. 
Table 2

Right paw entry (RPE) and preferred paw entry (PPE) correlations for the five reaching sessions

\begin{tabular}{|c|c|c|c|c|c|}
\hline & \multicolumn{5}{|c|}{ RPE correlations } \\
\hline & SN1 & SN1V & IND & $\mathrm{SN} 2$ & SW \\
\hline \multicolumn{6}{|c|}{ PPE correlations } \\
\hline SN1 & & $0.967 * * *$ & $0.873^{* * *}$ & $0.932 * * *$ & $0.858^{* * *}$ \\
\hline SN1V & $0.864 * * *$ & & $0.880 * * *$ & $0.937 * * *$ & $0.880^{* * *}$ \\
\hline IND & $0.473^{*}$ & $0.649^{* *}$ & & $0.927 * * *$ & $0.803^{* * *}$ \\
\hline SN2 & $0.765^{* * *}$ & $0.843^{* * *}$ & $0.516^{* *}$ & & $0.943^{* * *}$ \\
\hline SW & $0.666^{* * *}$ & $0.778^{* * *}$ & $0.706^{* * *}$ & $0.816^{* * *}$ & \\
\hline
\end{tabular}

RPE correlations are shown in the top-right array of the correlation matrix, and PPE correlations appear at the bottom left.

$* P<0.05, * * P<0.01$ and $* * * P<0.001$.

Abbreviations are as for Table 1.

Correlations for the RPE and PPE scores from all the sessions are presented in Table 2, and are all highly significant. In particular, there were very high correlations between session SN1, the first standard protocol session, and the videotaped data of that session (SN1V) for which the first 50 reaches with each paw were noted. Therefore, the procedure of observing several mice at once does not seem to bias the data.

\subsubsection{Degree of paw preference}

Notwithstanding the high correlations noted above, the distributions of scores for the PPE variable were quite different among the five sessions. Preliminary analysis revealed that the distributions of PPE scores for the standard protocol sessions (every session except IND) were very similar but differed substantially from that of the IND session. We therefore obtained a mean frequency distribution of PPE scores for the four standard sessions and compared this to the PPE frequency distribution for the IND session using the KolmogorovSmirnov two-sample test [21], the result of which was significant $\left(\chi^{2}(2)=10.07, P<0.01\right)$. The two cumulative relative frequency distributions are shown in Fig. 2. As can be seen from this figure, mice tended to be more lateralized for the IND session wherein independent reaches were recorded.

We also performed parametric tests on the transformed PPE scores. These were obtained by first transforming the RPE scores using a logit procedure in order to attenuate problems of nonnormality and range restriction $[14,22]$ :

$\mathrm{LRPE}=0.5 \ln \frac{\mathrm{RPE}+1 / 6}{(50-\mathrm{RPE})+1 / 6}$

We then obtained the absolute value of LRPE and performed the repeated-measures ANOVA on these (LPPE) values:

$$
\mathrm{LPPE}=|\mathrm{LRPE}|
$$

The results of the ANOVA were highly significant $(F(4,92)=8.84, P<0.001)$, which was a result of the discrepancy between the mean LPPE for the IND session, and those for the standard protocol sessions. These means, and their standard errors, are shown in Fig. 3.

A similarly discrepant result for the IND session was obtained when we tabulated the numbers of mice in each session that would be considered to be ambilateral by a criterion based on the binomial test. In 50 reaches, to be considered truly right-pawed, a mouse should reach at least 33 times with the right paw. Similarly, a left-pawed mouse should not reach with its right paw more than 17 times out of 50. This means that a mouse could be considered ambilateral with a PPE score of less than or equal to 7. Only one mouse met this criterion in the IND session, whereas between three and seven mice were thus classified for the four standardprotocol sessions.

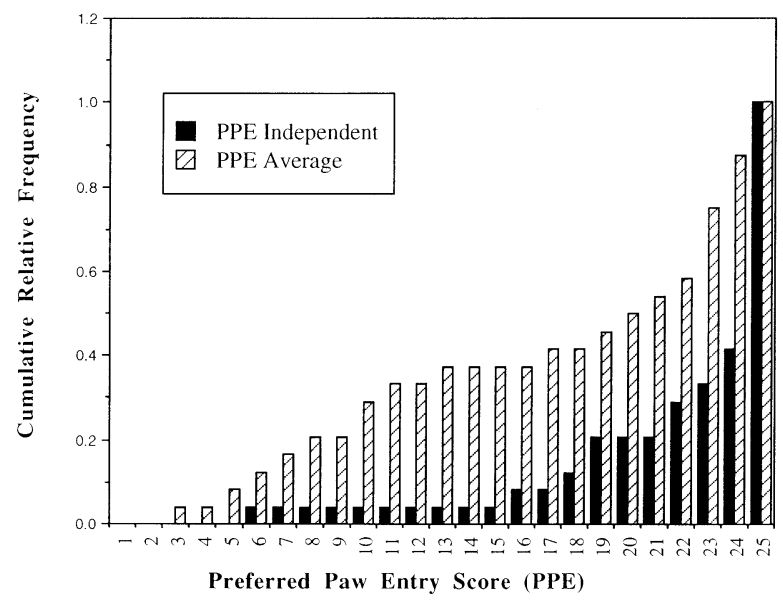

Fig. 2. Comparison of the cumulative frequency distributions for the preferred paw entry (PPE) scores on the independent-reaches test and the average of for the four standard-testing sessions in Study 1 (C57BL/6J mice). 


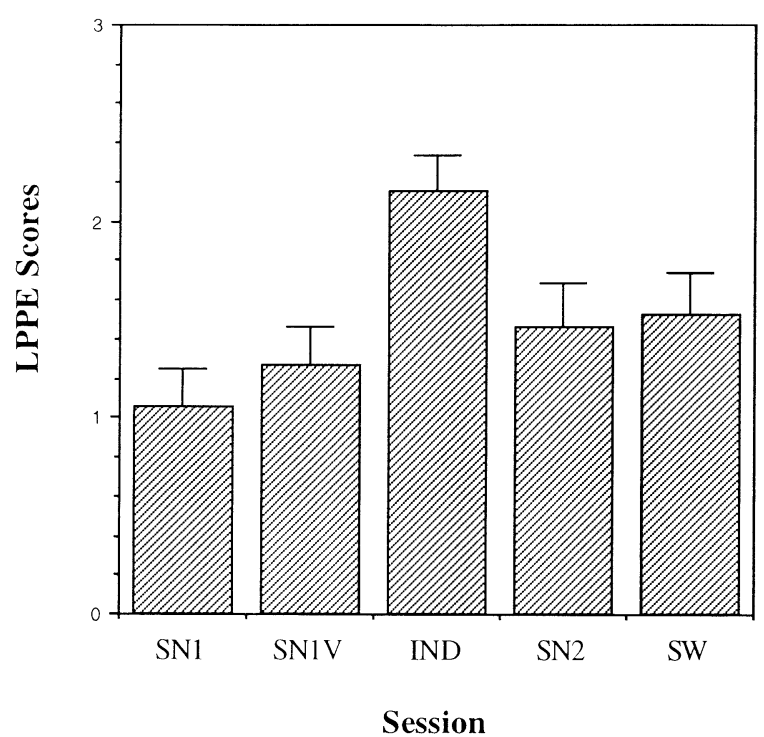

Fig. 3. The mean LPPE scores (see text for formula) for the paw preference testing sessions in Study 1 (C57BL/6J mice). Abbreviations: SN1, first standard protocol test with narrow tube; SN1V, videotaped data for first 50 reaches of SN1; IND, independentreaches protocol; SN2, second test using standard protocol, narrow tube; SW, standard protocol, wide tube.

In agreement with the high correlations between the SN1 and SN1V sessions, a matched-pairs $t$-test between the two LPPE measures was not significant $(t(23)=$ $1.67, P>0.05$, two-tailed).

\subsubsection{Practise effects and tube width}

Also of interest with respect to the PPE scores was the comparison of the two standard protocol sessions using the narrow tubes: sessions SN1 and S2, between which there was a period of 5 weeks when the animals performed the 50 independent reaches. A matched-pairs $t$-test on the mean difference between the LPPE scores for SN1 and S2 revealed a significant effect, with LPPE scores increasing from the first to the second session $(t(23)=2.23, P<0.05,2$-tailed) (see Fig. 3).

To assess the effects of tube width, we performed a matched-groups $t$-test on the difference between the mean of the LPPE scores for SN1 and SN2 (the two tests using the standard protocol with the narrow tube) and the LPPE mean score for SW (wide tube). The effect was not significant $(t(23)=1.54, P>0.05$, twotailed).

\subsection{Discussion}

The correlations between the SN1 session and the videotaped version of that session, SN1V, for RPE and PPE scores were very high (see Table 2). Furthermore, there were no differences in classifications when were dichotomized as either right- or left-preferent. It would seem, then, that observing any 50 reaches of mice during a testing session would lead one to draw essentially the same conclusions as would observing the first 50 consecutive reaches of each animal. This is important to know because of the extremely labour-intensive nature of the testing procedure.

The results of the runs tests on the consecutive reaches (videotaped) data give no support for the hypothesis that the reaches for the majority of the mice are not independent. Therefore, because the usual procedure involves random sampling from the consecutive sequence of reaches, there is no reason to question the independence of these data.

It is paradoxical, therefore, that the main finding was that the $\mathrm{C} 57 \mathrm{BL} / 6 \mathrm{~J}$ mice showed a greater degree of paw preference when tested under the independent-reaches condition than when tested in the standard manner, when all 50 reaches were recorded at one sitting. Because results from the standard protocol situation do not provide evidence to reject the hypothesis of independence of reaches, the independence per se of the individual-reaches protocol cannot provide the explanation for the differences observed, and other explanations must be investigated. In the standard situation, the mice often reach several times in quick succession with one paw, and it is possible that fatigue of the muscles involved in performing the reaching movement could account for these differences. For example, the right arm of a strongly right-preferent mouse may become tired after several reaches, and the animal may try out the left paw once or twice, until its right arm is rested and ready to reach for more food.

Another possible explanation for the discrepancy might be the stress of the handling involved with the IND protocol (Carlson, personal communication 1995). Because animals might be considered to be more stressed at the beginning of a testing session, it could be that the IND sessions, in which only the first reach was recorded, might have involved more stress than did the standard sessions. If this were the case, then, animals that were not very strongly lateralized (those for whom there was a non-neglible probability that a reach would be made with the non-preferred paw) would all be expected, at the beginning of a standard session, to reach with the paw that was concordant with their eventual classification. An examination of the first reaches of SN1, which was videotaped, revealed that as many of these animals' first reaches were not concordant as were concordant with their pawedness classification. Therefore, these results do not provide support for the stress explanation.

Food deprivation has been shown to influence rotatory behaviour in rats through its effects on prefrontal cortical systems that modulate the nigrostriatal system [23]. It is unlikely, however, that food deprivation played a role in the effects seen in the present experiment. For one thing, although all mice were food-de- 
Table 3

Study 2 means for raw RPE and PPE scores and numbers of mice classified as right- and left-pawed

\begin{tabular}{|c|c|c|c|c|c|c|c|}
\hline \multirow[t]{3}{*}{ Testing order } & \multirow[t]{3}{*}{$n$} & \multicolumn{6}{|c|}{ Testing protocol } \\
\hline & & \multicolumn{3}{|l|}{ Standard } & \multicolumn{3}{|l|}{ Independent } \\
\hline & & RPE (S.D.) & PPE (S.D.) & $\mathrm{R} / \mathrm{L}$ & RPE (S.D.) & PPE (S.D.) & $\mathrm{R} / \mathrm{L}$ \\
\hline SI & 16 & $19.4(17.9)$ & $16.6(7.6)$ & $7 / 9$ & $17.6(22.9)$ & 22.9 (4.9) & $5 / 11$ \\
\hline IS & 14 & $19.6(23.9)$ & $22.8(6.6)$ & $5 / 8^{\mathrm{a}}$ & $21.1(22.9)$ & $21.4(6.7)$ & $5 / 9$ \\
\hline
\end{tabular}

a One animal in the IS group had an RPE score of 25 on the standard-protocol test, so was not classified as R or L.

prived for the SN1 session, and some were also deprived for some of the independent-reaches tests, none was deprived for the final $\mathrm{SW}$ session. If food deprivation had been an important factor, one would not have expected to have observed the high correlations between the SW and the other sessions, in which most animals were food-deprived. Furthermore, there is evidence that paw preference and rotatory preference are probably subserved by different systems in the mouse [17].

There was a small but significant increase in the degree of paw preference between SN1 and SN2, indicating that mice become more lateralized with practise, a result that has been noted by other investigators $[10,24]$. This effect was too small to account for the protocol differences noted above, however. Although the effect of tube size in this study was confounded with effects of practise, it appears that there was very little difference between the results of the test when the wide as opposed to the narrow feeding tube was used. This result would argue against the fatigue hypothesis, in the sense that one might have expected fatigue to play a larger role in the narrow-tube than in the wide-tube situation.

One problem with the first study was that, for each mouse, the independent-reaches test always followed the testing by the standard protocol. Study 2 was undertaken to determine whether the effect of protocol observed in Study 1 would be observed with another inbred strain, and to counterbalance the order of the standard and independent-reaches tests.

\section{Study 2}

\subsection{Methods}

\subsubsection{Subjects}

The subjects for Study 2 were 16 male and 14 female pigmented congenic BALB/c mice (C.B6 $-+^{\mathrm{c}} /+^{\mathrm{c}}$ (N20); see [14] for details of the ancestry). They were between 10 and 12 weeks of age at the beginning of the study and were maintained under the same housing conditions as described in Study 1.

\subsubsection{Paw preference testing}

Each mouse was given the Collins test under two conditions: S (Standard condition, in which fifty reaches are recorded in one session), and I (Independent-reaches condition, as described in Study 1. The first reach is recorded on two separate occasions each day for 5 weeks). Sixteen animals (eight males, eight females) received the $\mathrm{S}$ protocol before the I protocol and for the remaining fourteen animals (eight males, six females) the order of testing was reversed. The standard, narrow tube was used for all testing. The animals for which I preceded S (IS) were food-deprived every afternoon for the subsequent day's I testing, and were also deprived before the S testing. The S-first group (SI) was food-deprived for the $\mathrm{S}$ test, but not for the subsequent I testing.

\subsection{Results}

\subsubsection{Direction of paw preference}

As in Study 1, the data were examined to determine the consistency of pawedness classification between the $\mathrm{S}$ and I testing sessions. Twenty-five of the animals were consistently classified over the two tests. One animal from the IS group had an RPE score of 25 on the $\mathrm{S}$ test, so was not classified as left- or as rightpawed. Four of 16 SI animals had inconsistent pawedness classifications on the $\mathrm{S}$ test and the I test but none of the 13 IS animals was discordantly classified on the two tests. Mean scores for the RPE and PPE measures, as well as the numbers of mice classified as right- and left-pawed for each of the tests are shown in Table 3. Correlations between the $\mathrm{S}$ and I protocols for RPE and PPE scores are shown in Table 4.

\subsubsection{Degree of paw preference}

Preliminary analyses showed that there were no sex effects, so sexes were pooled for further analyses. A repeated-measures ANOVA on the LPPE scores (the $\log$ transform of PPE, as for Study 1) with order of testing as the between-subjects factor and protocol as the within-subjects factor revealed a significant main effect of order $(F(1,28)=5.17, P<.05)$ and a significant protocol by interaction $(F(1,28)=17.28$, 
Table 4

Correlations for direction and degree measures between the standard protocol and independent-reaches protocols in Study 2

\begin{tabular}{llll}
\hline & All $(n=30)$ & $\begin{array}{l}\text { SI order }(n= \\
16)\end{array}$ & $\begin{array}{l}\text { IS order }(n= \\
14)\end{array}$ \\
\hline RPE score & $0.79^{* *}$ & $0.67^{* *}$ & $0.92^{* *}$ \\
PPE score & 0.16 & $0.55^{*}$ & -0.07 \\
$\begin{array}{l}\text { Left/right direc- } \\
\text { tion }\end{array}$ & $0.90^{* *}$ & $0.74^{* *}$ & $1.0^{* *}$ \\
\hline
\end{tabular}

${ }^{\text {a }}$ Cosine- $\pi$ tetrachoric correlations (for the IS order, this correlation is based on the 13 animals that could be classified as left- or right-preferent).

$* P<0.05$ and $* * P<0.01$

$P<.001$ ) (see Fig. 4). When mice were given the independent-reaches test first, they had larger mean PPE scores than did the standard-first group (Mean PPE scores: $I S=22.1 ; \quad S I=19.8$ ). As can be seen from Fig. 4, the significant interaction resulted from the increase in PPE from the first to the second test for the SI group, and the lack of difference (consistently high) between PPE scores on the two tests for the IS group. Although the main effect of test was not significant in this analysis, a matched-pairs $t$-test performed on the data from the SI group (LPPE scores) was significant $(t(15)=-4.71, P<.01)$, indicating that naïve mice, if given the standard Collins protocol first, exhibited significantly greater lateralization when subsequently tested by the independentreaches method. This result is in agreement with results from Study 1.

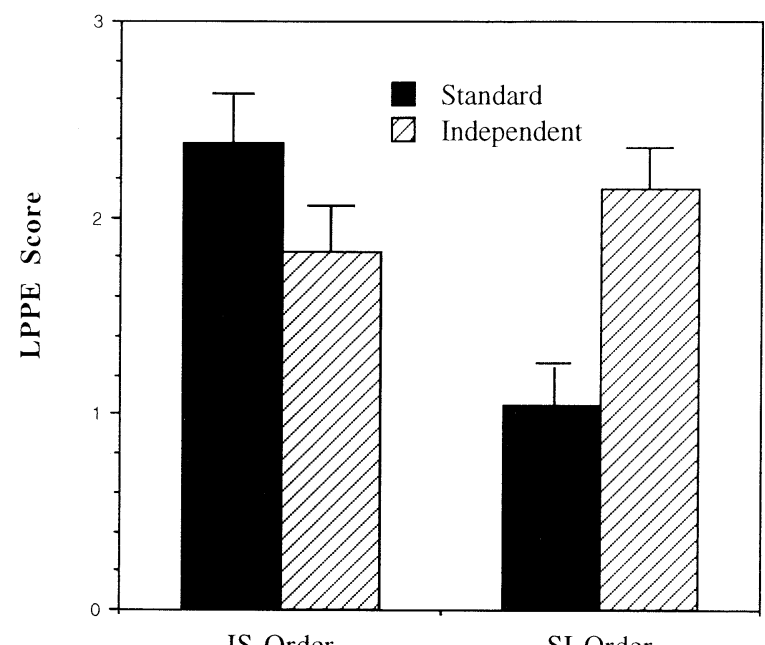

Order

Fig. 4. The mean LPPE scores for Study 2 (BALB mice). Abbreviations: IS independent-reaches protocol first, followed by standard protocol; SI Order, standard protocol first, followed by independent-reaches protocol.
Table 5

Comparison of Study 1 and Study 2 LPPE mean scores (S.D.)

\begin{tabular}{lllll}
\hline & $\begin{array}{l}\text { Mouse } \\
\text { strain }\end{array}$ & Standard & $\begin{array}{l}\text { Indepen- } \\
\text { dent }\end{array}$ & Standard \\
\hline $\begin{array}{c}\text { Study 1 } \\
\begin{array}{c}\text { Study 2 IS } \\
\text { order }\end{array}\end{array}$ & BAL7 & $1.06(0.92)$ & $2.16(0.90)$ & $1.46(1.1)$ \\
$\begin{array}{c}\text { Study 2 SI } \\
\text { order }\end{array}$ & BALB & $1.04(0.72)$ & $2.15(0.83)$ & \\
\hline
\end{tabular}

\subsection{Discussion}

Mice of the inbred BALB strain have a defect of the corpus callosum such that some animals have a midline callosal area that is much reduced and some animals have a complete lack of fibres crossing midplane [25]. It would seem reasonable, therefore, that mice of this strain might exhibit anomalous paw-preference behaviour based on the extent of their callosal deficit. However, a previous study with albino BALB mice [26], and one from this laboratory using the same pigmented BALB strain used in Study 2 [14] both failed to find evidence of a relation between midline callosal area and degree of paw preference as measured by the Collins test.

Results from Study 2 provided a partial replication of the first study for the difference between the PPE scores for the $\mathrm{S}$ and the I paw-preference testing protocols when the order of testing was the $\mathrm{S}$ protocol preceding the I protocol. Furthermore, this difference was observed in a different inbred strain of mice. It appears, however, as though the order in which the mice performed the tasks was an important factor in the determination of the degree of expressed paw preference. The mice that underwent the I protocol first subsequently exhibited a much stronger paw preference in the $\mathrm{S}$ condition than did naïve mice that performed the $\mathrm{S}$ protocol before the $\mathrm{I}$ protocol.

There is no obviously satisfactory explanation for the observed protocol by order interaction, but it was the case that all the mice in Study 2 showed a greater degree of preference in the second paw preference test than in the first, irrespective of the order of testing. It could be the case that BALB mice are exhibiting practise effects, rather larger than those observed in Study 1 for the C57 strain. If this is the case, however, the fatigue hypothesis put forward earlier as an explanation for the lower preference scores for the $\mathrm{S}$ protocol is not tenable, because IS animals were highly preferent for $\mathrm{S}$ testing in this study (see Table 5). 


\section{General discussion}

The results of Study 1 indicate that B6 mice appear to be more lateralized when tested using the independent-reaches protocol. In addition, analysis of the videotaped data indicated that four of the animals exhibited a lack of independence of reaches using the standard protocol. The second study replicated the increased manifest laterality shown using the independent-reaches method, and with BALB mice. In this study, however, the results indicated that the mice, when their first experience was with the independentreaches method, were as highly lateralized with the standard protocol as they were with the independentreaches protocol.

The independent-reaches protocol has the advantages of assuring independence of reaches and of being immune from the order effects that affect the standard protocol. It will be important in future work to investigate the nature of these order effects. Inherent in many human handedness preference questionnaires is the concept of the degree to which one is right- or left-preferent, and, indeed, there is some evidence that the degree of human hand preference may be hereditary [27]. If the mouse model is to be a useful one for the study of the development and nature of human handedness it will obviously be important to understand the various factors that govern expressed pawedness in the mouse.

\section{Acknowledgements}

Revisions to this paper were made while MBBF was on sabbatical leave at the University of York, UK. She is most appreciative of the use of their facilities, as well as the support of many members of the Psychology Department at York. The very helpful comments of two reviewers are also gratefully acknowledged, as are preliminary discussions with Pat Wainwright and Chris McManus. The authors would also like to thank Val Hay, Tami Free and Kerry-Ann Maddix for their help with the paw-preference testing, and Tracy Cocivera and Gina Grimshaw for scoring the videotaped session. The Department of Kinesiology, University of Waterloo, very kindly made available the equipment to analyse the videotapes. We are indebted to Nancy Gibson for her excellent technical skills and her conscientious care of the animals. This research was supported in part by a grant from the Natural Sciences and Engineering Research Council of Canada to MPB.

\section{References}

[1] Bradshaw, J.L. and Rogers, L.J., The Evolution of Lateral Asymmetries, Language, Tool Use, and Intellect, 1993, Academic Press, San Diego, 463pp.
[2] Fagot, J. and Vauclair, J., Manual laterality in nonhuman primates: A distinction between handedness and manual specialization, Psychol. Bull., 109 (1991) 76-89.

[3] Glick, S.D., Cerebral Lateralization in Nonhuman Species, 1985, Academic Press, Orlando, FL, 288pp.

[4] McManus, I.C. and Bryden, M.P., The neurobiology of handedness, language and cerebral dominance: A model for the molecular genetics of behaviour. In M.H. Johnson (Ed.), Brain Development and Cognition: A Reader, 1993, Blackwells, Oxford, pp. $679-702$.

[5] Yokoyama, T., Copeland, N.G., Jenkins, N.A., Montgomery, C.A., Elder, F.F.B. and Overbeek, P.A., Reversal of left-right asymmetry: A situs inversus mutation, Science, 260 (1993) 679682.

[6] Collins, R.L., The sound of one paw clapping: An inquiry into the origin of left-handedness. In D. Lindzey and D. Thiessen (Eds.), Contributions to Behavior-Genetic Analysis: The mouse as a Prototype, 1970, Appleton-Century-Crofts, New York, pp. 115-136.

[7] Collins, R.L., On the inheritance of handedness II. Selection for sinistrality in mice, J. Hered., 60 (1969) 117-119.

[8] Collins, R.L., On the inheritance of direction and degree of asymmetry. In S.D. Glick (Ed.), Cerebral Lateralization in Nonhuman Species, 1985, Academic Press, Orlando, FL, pp. 41-71.

[9] Signore, P., Chaoui, M., Nosten-Bertrand, M., Perez-Diaz, F. and Marchaland, C., Handedness in mice: Comparison across eleven inbred strains, Behav. Genet., 21 (1991) 421-429.

[10] Alonso, J., Castellano, M.A. and Rodriguez, M., Behavioral lateralization in rats: Prenatal stress effects on sex differences, Brain Res., 539 (1991) 45-50.

[11] Waters, N.S. and Denenberg, V.H., Analysis of two measures of paw preference in a large populaton of inbred mice, Behav. Brain Res., 63 (1994) 195-204.

[12] Mutsaers, L.M., Is an increase in lateral preference the means by which environmental enrichment improves spatial learning? Honours BSc. Thesis, 1995, University of Waterloo, Waterloo, ON, Canada.

[13] Biddle, F.G., Coffaro, C.M., Ziehr, J.E. and Eales, B.A., Genetic variation in paw preference (handedness) in the mouse, Genome, 36 (1993) 935-943.

[14] Bulman-Fleming, B., Wainwright, P. and Collins, R.L., The effects of early experience on callosal development and functional lateralization in pigmented $\mathrm{BALB} / \mathrm{c}$ mice, Behav. Brain Res., 50 (1992) 31-42.

[15] Gerritsen, J.A. and Biddle, F.G., The SWV and C57BL/6 inbred strains exhibit the same difference in lateralization of handedness as the selected LO and HI lines, Mouse Genome, 90 (1992) $90-92$.

[16] Neveu, P.J., Barnéoud, P., Vitiello, S., Betancur, C. and Le Moal, M., Brain modulation of the immune system: Association between lymphocyte responsiveness and paw preference in mice, Brain Res., 457 (1988) 392-394.

[17] Nielsen, D.M., Visker, K.E., Cunningham, R.W., Keller Jr., R.W., Glick, S.D., and Carlson, J.N., Paw preference, rotation, and dopamine function in Collins' HI and LO mouse strains, Physiol. Behav., (1997) in press.

[18] Schmidt, S.L., Manhães. A.C. and de Moraes, V.Z., The effects of total and partial callosal agenesis on the development of paw preference performance in the $\mathrm{BALB} / \mathrm{CCF}$ mouse, Brain Res., 545 (1991) $123-130$.

[19] Waters, N.S. and Denenberg, V.H., A measure of lateral paw preference in the mouse, Physiol. Behav., 50 (1991) 853-856.

[20] Siegel, S. and Castellan Jr., N.J., Nonparametric Statistics for the Behavioral Sciences, 2nd edn., 1988, McGraw-Hill, New York, 399pp.

[21] Hays, W.L., Statistics, 4th edn., 1988, Holt, Rinehart and Winston, Fort Worth, 1029pp. 
[22] Cassells, B., Collins, R.L. and Wahlsten, D., Path analysis of sex difference, forebrain commissure area and brain size in relation to degree of laterality in selectively bred mice, Brain Res., 529 (1990) 50-56.

[23] Carlson, J.N., Glick, S.D., Hinds, P.A. and Baird, J.L., Food deprivation alters dopamine utilization in the rat prefrontal cortex and asymmetrically alters amphetamine-induced rotational behavior, Brain Res., 454 (1988) 373-377.

[24] Collins, R.L., When left-handed mice live in right-handed worlds, Science, 187 (1975) 181-184.

[25] Wahlsten, D., Genetic and developmental defects of the mouse corpus callosum, Experientia, 45 (1989) 828-838.

[26] Ward, R., Tremblay, L. and Lassonde, M., The relationship between callosal variation and lateralization in mice is genotype dependent, Brain Res., 424 (1987) 84-88.

[27] Bryden, M.P., Handedness and cerebral organization: Data from clinical and normal populations. In D. Ottoson (Ed.), Duality and Unity of the Brain, 1987, MacMillan, London, pp. 55-70. 